\title{
Paleopathology of malignant tumours supports the concept of human vulnerability to cancer
}

\author{
Andreas G. Nerlich* and Beatrice E. Bachmeier ${ }^{*}$ \\ * Department of Pathology, Academic Hospital Munich-Bogenhausen, \\ Englschalkingerstr. 77, D-81925 Munich, Germany. \\ ${ }^{\ddagger}$ Department of Clinical Chemistry and Clinical Biochemistry, Ludwig-Maximilians- \\ University Munich, Nussbaumstr. 20, D-80336 Munich, Germany.
}

Correspondence to A.G.N. e-mail: Andreas.Nerlich@extern.Irz-muenchen.de

We read with great interest the Perspective opinion of Mel Greaves ${ }^{1}$ about Darwinian medicine, including his concept of the increased vulnerability of the human species to cancer. However, there is one small aspect that seems to have escaped the author's notice. Although the author states that "medical reports and paleopathological studies ... do tell us that cancer existed 2,000 years or more ... but do not provide cancer rates", such paleoepidemiological studies that indicate malignant tumour rates in historic populations, do in fact exist ${ }^{2,3}$. We have recently shown in two ancient populations - one from Egypt dating from about 1500-500 BC, one from medieval to modern Europe dating from about AD 14001800 - that the rates of malignant tumours on an age- and sex-adjusted population basis are comparable in both populations, and compare well with a reference population from modern England dating from AD 1900-1905 (REF. 4). The population from England that served as a basis for the estimation model also takes the rates of osseous involvement into account $^{4}$, so that malignant tumour frequencies become comparable even in the dry bone conditions that are usually seen in paleopathological studies. Both of our study populations comprised several hundred to thousand mummies or skeletons (in the Egyptian material 905 individuals, in the German material at least 2547 individuals), and as a result we consider the observed tumour frequencies to be representative, although the absolute tumour numbers are still low.

Our study supports Greaves' opinion that malignant tumours are not just a recent problem, but that they were prevalent 4,500 years or more ago - provided that individuals reached a sufficient age for any tumour-promoting defect to be clinically manifest. Although these data are preliminary, they support Greaves' concept that malignant tumours are inherent to humans, with implications for the Darwinian model of human evolution.

1. Greaves, M. Darwinian medicine: a case for cancer. Nature Rev. Cancer $\mathbf{7}$, 213-221 (2007).

2. Zink, A. et al. Malignant tumours in an ancient Egyptian population. Anticancer Res. 19, 4273-4278 (1999)

3. Nerlich, A. G., Rohrbach, H., Bachmeier, B. E. \& Zink, A. Malignant tumours in two ancient populations: an approach to historical tumour epidemiology.

Oncol. Rep. 16, 197-202 (2006).

4. Waldron, T. What was the prevalence of malignant disease in the past? Int. J. Osteoarcheol. 6, 463-470 (1996). 